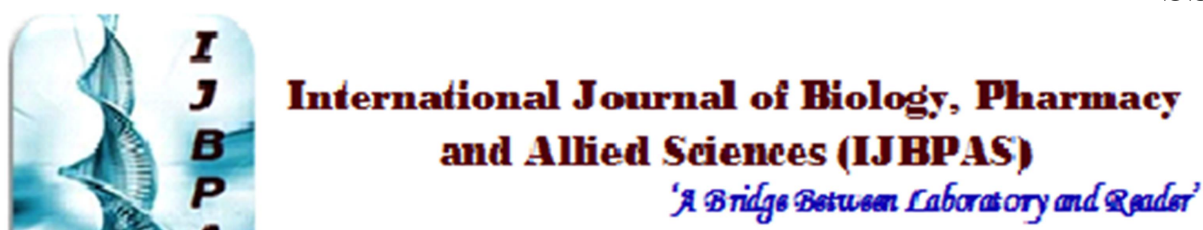

\author{
Www.ijbpas.com
}

\title{
RPGT-TRANSMISSION MODEL TO CONTROL COVID-19 EPIDEMIC
}

\author{
KULDEEP SINGH $^{1 *}$ AND RAKESH YADAV ${ }^{2}$
}

1, 2: Department of Mathematics, Lovely Professional University, Phagwara, Punjab, India

*Corresponding Author: Dr. Kuldeep Singh; E Mail: kuldeep.41900036@Ipu.in

Received $19^{\text {th }}$ Aug. 2021; Revised 20 ${ }^{\text {th }}$ Sept. 2021; Accepted $29^{\text {th }}$ Oct. 2021; Available online $1^{\text {st }}$ Dec. 2021

https://doi.org/10.31032/IJBPAS/2021/10.12.2023

\begin{abstract}
The COVID-19 outbreak is a sharp reminder that pandemics, as other rarely happening catastrophes, have occurred before and will keep on reoccurring. Transmission models are helpful to comprehend the behaviour of contamination when it enters a community and explores under which conditions it will be treated or processed. Subsequently, there are few treatments in India dealing with an intense issue not just in India the comparable situation is seen all through the globe. To start with, the detailing of the model is proposed; at that point, the positivity of the model is discussed. Using RPGT, Transition Diagram of the system and expressions for Transition Probability and Mean Sojourn Times, Path Probabilities, Mean time to Epidemic affected $\left(\mathrm{T}_{0}\right)$, Average Healthy Time $\left(\mathrm{A}_{0}\right)$, and Recovery Period $\left(\mathrm{B}_{0}\right)$ are derived followed by illustrations. Tables and graphs are drawn followed by analysis.
\end{abstract}

\section{Keywords: RPGT, Coronaviruses, Respiratory Syndrome, Sanitization}

\section{INTRODUCTION}

The COVID-19 first showed up in Wuhan city, and because of its high transmission rate, the infection has been quickly spreading everywhere in the world. Coronaviruses are a huge group of zoonotic viruses, i.e., these are communicated from animals to people, and that cause side effects going from the basic virus to more serious illnesses like Middle East Respiratory Syndrome which is sent from dromedary to people and serious intense respiratory syndrome which is communicated from civet to people. A few known covid-19 variants that have not yet contaminated people are flowing in certain creatures. The current episode has had seriously ill affected health and financial outcomes across the globe, and it does not appear as though any nation will be unaffected. This not just has ramifications for the health and economy; all of society is influenced, which has prompted emotional changes in how organizations and buyers to act. Researchers in all walks have been following 
the spread of the virus, have activated to speed inventive diagnostics, and are chipping away at various antibodies to ensure against covid-19. [2] studied the mathematical model for covid19 containing confinement class. [4] determined the clinical highlights of covid-19 and contemplated the momentary aftereffects of 18 patients and 102 patients with covid-19 in escalated care units. [6] inspected that few local health facilities reported a group of patients with pneumonia for obscure reasons connected to the seafood and wholesale wet animal market in Wuhan, Hubei Province, China. The Place for Disease Control and Prevention dispatched a fast reaction group to help wellbeing experts in Hubei Province and Wuhan City and lead an epidemiological investigation to find the wellspring of pneumonia groups. This prompted a portrayal of another Coronavirus found in examples of pneumonia patients toward the start of the pestilence. [7] examined the Extended SEIR Model with Vaccination for Forecasting the covid-19 Pandemic in Saudi Arabia using an Ensemble Kalman Filter. [8] talked about the numerical models which have been created to examine the transmission elements of covid- 19 . Nonetheless, these models experience the ill effects of different wellsprings of vulnerabilities, because of the fragmented depiction of the natural cycles overseeing the illness spread, and furthermore because of some elaborate boundaries being ineffectively known. One approach to relieve these vulnerabilities is to oblige plague estimating models with accessible information. [3] discussed Forecasting the size of covid-19 in
India utilizing the ARIMA Model. [1] examined the introduction on utilizing math to comprehend covid-19 dynamics: Modelling, examination, and recreations. [10] dissected the washing unit in a paper factory utilizing RPGT. [11] examined the bread-making system and considered the behaviour analysis. [5] studied the SEIRD model for covid-19. In this paper, parameters are observed to make forecasts about deaths and the number of cases. [12] contemplated the benefit of an edible oil refinery plant. [9] discussed the Polynomials Approximation Model for the estimation of the number of infected peoples in India

Using Regenerative Point Graphical technique (RPGT) Transition Diagram of the system, expressions for Transition Probability and Mean Sojourn Times, Path Probabilities, Mean time to Epidemic affected $\left(\mathrm{T}_{0}\right)$, Average Healthy Time $\left(\mathrm{A}_{0}\right)$, and Recovery Period $\left(\mathrm{B}_{0}\right)$ are derived followed by illustrations. Tables and graphs are drawn followed by analysis.

Notations

1.1.1. E: F: G: H :: Sanitization: Wearing Mask: Social Distancing: Quarantine State

1.1.2. $\mathrm{e}, \mathrm{f}, \mathrm{g}, \mathrm{h}::$ denotes corresponding failure in observing precautions respectively.

1.1.3. $\mathrm{H}_{1}, \mathrm{H}_{2}$ :: Signify home isolation, Lockdown including micro lockdown which are standby to Quarantine State $(\mathrm{H})$

1.1.4. $n_{i} / m_{i}(i=1,2,3,4)::$ recovery/causing pandemic mean rates due to non-wearing mask, not keeping social distance, non-observing sanitization, non-observing Quarantine properly.

1.1.5. $f_{j}$ : Fuzziness measure of the $\mathrm{j}$-state. 


\subsection{ASSUMPTIONS}

\subsubsection{Affected/recovery rates are}

exponentially distributed and are independent.

1.2.2. On recovery an affected person is as healthy as not affected due to covid-19.

1.2.3. Recovery and being affected rates are independent identically distributed.

1.2.4. When a person is tested covid-19

positive no further covid-19 affection is

possible.

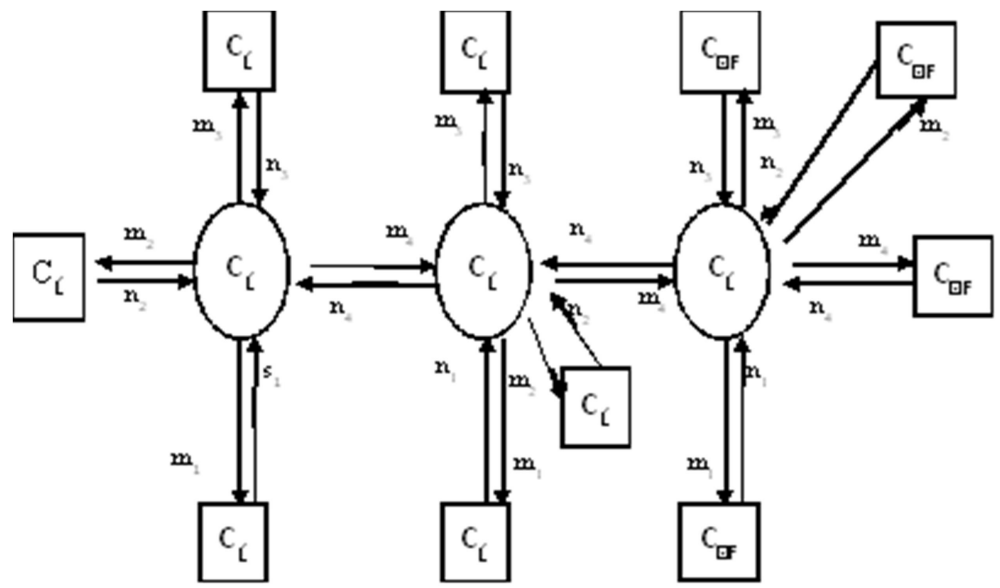

Figure 1: Transition Diagram of the system
$\mathrm{C}_{1}=\mathrm{EFGH}$,
$\mathrm{C}_{2}=\mathrm{eFGH}$,
$\mathrm{C}_{3}=\mathrm{EfGH}$,
$\mathrm{C}_{4}=\mathrm{EFgH}$,
$\mathrm{C}_{5}=\mathrm{EFGH}_{1}$,
$\mathrm{C}_{6}=\mathrm{eFGH}_{1}$,
$\mathrm{C}_{7}=\mathrm{EfGH}_{1}$,
$\mathrm{C}_{8}=\mathrm{EFgH}_{1}$,
$\mathrm{C}_{9}=\mathrm{EFGH}_{2}$,
$\mathrm{C}_{10}=\mathrm{eFGH}_{2}$,
$\mathrm{C}_{11}=\mathrm{EfGH}_{2}$,
$\mathrm{C}_{12}=\mathrm{EFgH}_{2}$,
$\mathrm{C}_{13}=\mathrm{EFGh}$

\subsection{TRANSITION PROBABILITY AND MEAN SOJOURN TIMES}

$\mathrm{p}_{\mathrm{i}, \mathrm{j}}(\mathrm{t})$ : Probability distribution function from $\mathrm{i}$

to $\mathrm{j}$. $\mathrm{q}_{\mathrm{ij}}$ : Transition probability from $\mathrm{i}$ to $\mathrm{j}$. $\mathrm{q}_{\mathrm{i}, \mathrm{j}}=\mathrm{p}_{\mathrm{i}, \mathrm{j}} *(0)$; where $*$ designate Laplace transform

where $\quad \eta_{i}^{1}$ is waiting period for detection/ testing

Table 1: Transition Probabilities

\begin{tabular}{|c|c|}
\hline $\mathbf{p}_{\mathrm{i}, \mathrm{j}}(\mathbf{t})$ & $q_{i j}=p^{*}{ }_{i, j}(0)$ \\
\hline $\begin{array}{c}p_{1, i}(t)=m_{j} e^{-\left(m_{1}+m_{2}+m_{3}+m_{4}\right) t} \\
i=2,3,4,5 \& j=1,2,3,4\end{array}$ & $\begin{array}{c}q_{1, i}=m_{j} /\left(m_{1}+m_{2}+m_{3}+m_{4}\right) \\
i=2,3,4,5 \& j=1,2,3,4\end{array}$ \\
\hline$p_{2,1}=n_{1} e^{-n_{1} t}$ & $q_{2,1}=1$ \\
\hline$p_{3,1}=n_{2} e^{-n_{2} t}$ & $q_{3,1}=1$ \\
\hline$p_{4,1}=n_{3} e^{-n_{3} t}$ & $q_{4,1}=1$ \\
\hline $\begin{aligned} p_{5,1}(t) & =n_{4} e^{-\left(m_{1}+m_{2}+m_{3}+m_{4}+n_{4}\right) t} \\
p_{5, i}(t) & =m_{j} e^{-\left(m_{1}+m_{2}+m_{3}+m_{4}+n_{4}\right) t} \\
i & =6,7,8,9 \& j=1,2,3,4\end{aligned}$ & $\begin{array}{c}q_{5,1}=n_{4} /\left(m_{1}+m_{2}+m_{3}+m_{4}+n_{4}\right) \\
q_{5, i}=m_{j} /\left(m_{1}+m_{2}+m_{3}+m_{4}+n_{4}\right) \\
i=6,7,8,9 \& j=1,2,3,4\end{array}$ \\
\hline$p_{6,5}=n_{1} e^{-n_{1} t}$ & $q_{6,5}=1$ \\
\hline$p_{7,5}=n_{2} e^{-n_{2} t}$ & $q_{7,5}=1$ \\
\hline$p_{8,5}=n_{3} e^{-n_{3} t}$ & $q_{8,5}=1$ \\
\hline
\end{tabular}




\begin{tabular}{|c|c|}
\hline $\begin{array}{c}p_{9,5}(t)=n_{4} e^{-\left(m_{1}+m_{2}+m_{3}+m_{4}+n\right) t} \\
p_{9, i}(t)=m_{j} e^{-\left(m_{1}+m_{2}+m+m_{4}+n_{4}\right) t} \\
\quad \mathbf{i}=10,11,12,13 \& \mathbf{j}=1,2,3,4\end{array}$ & $\begin{array}{c}q_{9,5}=n_{4} /\left(r_{1}+r_{2}+r_{3}+r_{4}+n_{4}\right) \\
q_{9, i}=m_{j} /\left(m_{1}+m_{2}+m_{3}+m_{4}+n_{4}\right) \\
i=10,11,12,13 \& j=1,2,3,4\end{array}$ \\
\hline$p_{10,9}=s_{1} e^{-s_{1} t}$ & $q_{10,9}=1$ \\
\hline$p_{11,9}=s_{2} e^{-s_{2} t}$ & $q_{11,9}=1$ \\
\hline$p_{12,9}=s_{3} e^{-s_{3} t}$ & $q_{12,9}=1$ \\
\hline$p_{13,9}=s_{4} e^{-s_{4} t}$ & $q_{13,9}=1$ \\
\hline
\end{tabular}

\begin{tabular}{|c|c|} 
Table 2: Mean Sojourn Time \\
\begin{tabular}{|c|c|}
\hline $\mathrm{S}_{\mathrm{i}}(\mathrm{t})$ & $\eta_{\mathrm{i}}=\mathrm{S}_{\mathrm{i}}^{*}(\mathbf{0})$ \\
\hline$S_{1}(\mathrm{t})=e^{-\left(m_{1}+m_{2}+m_{3}+m_{4}\right) t}$ & $\eta_{1}=1 /\left(\mathbf{m}_{1}+\mathbf{m}_{2}+\mathbf{m}_{3}+\mathbf{m}_{4}\right)$ \\
\hline$S_{1+i}(t)=e^{-\mathbf{n}_{i} t}, i=1,2,3$ & $\eta_{1+\mathrm{i}}=1 / \mathbf{n}_{\mathrm{i}}, i=1,2,3$ \\
\hline$S_{i}(\mathrm{t})=e^{-\left(m_{1}+m_{2}+m_{3}+m_{4}+n_{4}\right) t}, i=$ & $\eta_{\mathrm{i}}=1 /\left(\mathbf{m}_{1}+\mathbf{m}_{2}+\mathbf{m}_{3}+\mathbf{m}_{4}+\mathbf{n}_{4}\right), i=5,9$ \\
5,9 & $\eta_{5+\mathrm{i}}=1 / \mathbf{n}_{\mathrm{i}}, i=1,2,3$ \\
\hline$S_{5+i}(t)=e^{-i t}, i=1,2,3$ & $\eta_{9+\mathrm{i}}=1 / \mathbf{n}_{\mathrm{i}}, i=1,2,3,4$ \\
\hline$S_{9+i}(t)=e^{-i t}, i=1,2,3,4$ &
\end{tabular}
\end{tabular}

From Table 2 the expressions for $\boldsymbol{\eta}_{1}, \boldsymbol{\eta}_{5}, \boldsymbol{\eta}_{9}$, it is observed that if rates for being covid-19 effective are higher, then the mean sojourn time for a person to remain healthy is very small, hence such an atmosphere should be created so that causes which lead to covid-19 affected should be the bare minimum and from the expressions for states when a person is covid19 affected i.e., $\boldsymbol{\eta}_{1+\mathrm{i}}$, where $\mathrm{i}=2,3,4,6,7,8$, $10,11,12,13$ if the values of recovery rates are higher, then the mean sojourn time for a human to remain covid-19 affected will be small, hence recovery rates should be maintained higher.

\section{EVALUATION OF PATH PROBABILITIES $\left(\mathbf{E}_{\mathrm{I}, \mathrm{J}}\right)$ :}

Applying RPGT and using ' 1 ' as the initial state of the framework: Transition probability factors of all reachable states from the initial (base) state ' $\xi$ ' $=$ ' 1 ' are: Probabilities from state ' 1 ' to various vertices are given as

$\mathrm{E}_{1,1}=1$

$E_{1, i}=q_{1, i} ;$ where $i=2,3,4$

$\mathrm{E}_{1,5}=\mathrm{q}_{1,5} /\left(1-\mathrm{q}_{5,6} \mathrm{q}_{6,}, 5\right)\left(1-\mathrm{q}_{5,7} \mathrm{q}_{7,5}\right)\left(1-\mathrm{q}_{5,8} \mathrm{q}_{8,5}\right)\{(1-$

$\left.\mathrm{q}_{5,9} \mathrm{q}_{9,5}\right) /\left(1-\mathrm{q}_{9,10} \mathrm{q}_{10,9}\right)\left(1-\mathrm{q}_{9,11} \mathrm{q}_{11,9}\right)$

$\left.\left(1-\mathrm{q}_{9,12} \mathrm{q}_{12,9}\right)\left(1-\mathrm{q}_{9,13} \mathrm{q}_{13,9}\right)\right\}$
$\mathrm{E}_{1, \mathrm{i}}=\mathrm{q}_{1,5} \mathrm{q}_{5, i} /\left(1-\mathrm{q}_{5,6} \mathrm{q}_{5,6}\right)\left(1-\mathrm{q}_{5,7} \mathrm{q}_{7,5}\right)\left(1-\mathrm{q}_{5,8} \mathrm{q}_{8,5}\right)\{(1-$

$\left.\mathrm{q}_{5,9} \mathrm{q}_{9,5}\right) /\left(1-\mathrm{q}_{9,10} \mathrm{q}_{10,9}\right)\left(1-\mathrm{q}_{9,11} \mathrm{q}_{11,9}\right)$

$\left.\left(1-\mathrm{q}_{9,12} \mathrm{q}_{12,9}\right)\left(1-\mathrm{q}_{9,13} \mathrm{q}_{13,9}\right)\right\} ; \mathrm{i}=6,7,8$.

$\mathrm{E}_{1,9}=\mathrm{q}_{1,5} \mathrm{q}_{5,9} /\left(1-\mathrm{q}_{5,6} \mathrm{q}_{6,5}\right)\left(1-\mathrm{q}_{5,7} \mathrm{q}_{7,5}\right)\left(1-\mathrm{q}_{5,8} \mathrm{q}_{8,5}\right)(1-$

$\left.\mathrm{q}_{9,10} \mathrm{q}_{10,9}\right)\left(1-\mathrm{q}_{9,11} \mathrm{q}_{11,9}\right)\left(1-\mathrm{q}_{9,12} \mathrm{q}_{12,9}\right)$

$\left(1-q_{9,13} q_{13}, \quad\right.$ 9) $\left\{\left(1-q_{5,9} q_{9,5}\right) /\left(1-q_{9,10} q_{10,9}\right)(1-\right.$

$\left.\left.\mathrm{q}_{9,11} \mathrm{q}_{11,9}\right)\left(1-\mathrm{q}_{9,12} \mathrm{q}_{12,9}\right)\left(1-\mathrm{q}_{9,13} \mathrm{q}_{13,9}\right)\right\}$

$\mathrm{E}_{1, \mathrm{i}}=\quad \mathrm{q}_{1,5} \mathrm{q}_{5,9} \mathrm{q}_{9, i} /\left(1-\mathrm{q}_{5,6} \mathrm{q}_{6,5}\right)\left(1-\mathrm{q}_{5,7} \mathrm{q}_{7,5}\right)(1-$

$\left.\mathrm{q}_{5,8} \mathrm{q}_{8,5}\right)\left(1-\mathrm{q}_{9,10} \mathrm{q}_{10,9}\right)\left(1-\mathrm{q}_{9,11} \mathrm{q}_{11,9}\right)\left(1-\mathrm{q}_{9,12} \mathrm{q}_{12,9}\right)$

$\left(1-\mathrm{q}_{9,13} \mathrm{q}_{13,}, \quad\right.$ 9) $\left\{\left(1-\mathrm{q}_{5,9} \mathrm{q}_{9,5}\right) /\left(1-\mathrm{q}_{9,10} \mathrm{q}_{10,9}\right)(1-\right.$

$\left.\left.\mathrm{q}_{9,11} \mathrm{q}_{11,9}\right)\left(1-\mathrm{q}_{9,12} \mathrm{q}_{12,9}\right)\left(1-\mathrm{q}_{9,13} \mathrm{q}_{13,9}\right)\right\}$;

where $\mathrm{i}=10,11,12,13$.

Probabilities from state ' 9 ' to different vertices are given as

$\mathrm{E}_{9,1}=\mathrm{q}_{9,5} \mathrm{q}_{5,1} /\left(1-\mathrm{q}_{5,6} \mathrm{q}_{6,5}\right)\left(1-\mathrm{q}_{5,7} \mathrm{q}_{7,5}\right)\left(1-\mathrm{q}_{5,8} \mathrm{q}_{8,5}\right)(1-$

$\left.\mathrm{q}_{1,2} \mathrm{q}_{2,1}\right)\left(1-\mathrm{q}_{1,3} \mathrm{q}_{3,1}\right)\left(1-\mathrm{q}_{1,4} \mathrm{q}_{4,1}\right)$

$\left\{\left(1-\mathrm{q}_{5,1} \mathrm{q}_{1,5}\right) /\left(1-\mathrm{q}_{1,2} \mathrm{q}_{2,1}\right)\left(1-\mathrm{q}_{1,3} \mathrm{q}_{3,1}\right)\left(1-\mathrm{q}_{1,4} \mathrm{q}_{4,1}\right)\right\}$

$\mathrm{E}_{9, \mathrm{i}}=\quad \mathrm{q}_{9,5} \mathrm{q}_{5,1} \mathrm{q}_{1, i} /\left(1-\mathrm{q}_{5,6} \mathrm{q}_{6,5}\right)\left(1-\mathrm{q}_{5,7} \mathrm{q}_{7,5}\right)(1-$

$\left.\mathrm{q}_{5,8} \mathrm{q}_{8,5}\right)\left(1-\mathrm{q}_{1,2} \mathrm{q}_{2,1}\right)\left(1-\mathrm{q}_{1,3} \mathrm{q}_{3,1}\right)\left(1-\mathrm{q}_{1,4} \mathrm{q}_{4,1}\right)$

$\left\{\left(1-\mathrm{q}_{5,1} \mathrm{q}_{1,5}\right) /\left(1-\mathrm{q}_{1,2} \mathrm{q}_{2,1}\right)\left(1-\mathrm{q}_{1,3} \mathrm{q}_{3,1}\right)\left(1-\mathrm{q}_{1,4} \mathrm{q}_{4,1}\right)\right\}$;

where $\mathrm{i}=2,3,4$.

$\mathrm{E}_{9,5}=\mathrm{q}_{9,5} /\left(1-\mathrm{q}_{5,6} \mathrm{q}_{6,5}\right)\left(1-\mathrm{q}_{5,7} \mathrm{q}_{7,5}\right)\left(1-\mathrm{q}_{5,8} \mathrm{q}_{8,5}\right)\{(1-$

$\left.\left.\mathrm{q}_{5,1} \mathrm{q}_{1,5}\right) /\left(1-\mathrm{q}_{1,2} \mathrm{q}_{2,1}\right)\left(1-\mathrm{q}_{1,3} \mathrm{q}_{3,1}\right)\left(1-\mathrm{q}_{1,4} \mathrm{q}_{4,1}\right)\right\}$

$\mathrm{E}_{9, \mathrm{i}}=\mathrm{q}_{9,5} \mathrm{q}_{5, i} /\left(1-\mathrm{q}_{5,6} \mathrm{q}_{6,5}\right)\left(1-\mathrm{q}_{5,7} \mathrm{q}_{7,5}\right)\left(1-\mathrm{q}_{5,8} \mathrm{q}_{8,5}\right)\{(1-$

$\left.\left.\mathrm{q}_{5,1} \mathrm{q}_{1,5}\right) /\left(1-\mathrm{q}_{1,2} \mathrm{q}_{2,1}\right)\left(1-\mathrm{q}_{1,3} \mathrm{q}_{3,1}\right)\left(1-\mathrm{q}_{1,4} \mathrm{q}_{4,1}\right)\right\}$;

Where $\mathrm{i}=6,7,8$. 
$\mathrm{E}_{9,9}=1$

$E_{9, \mathrm{i}}=\mathrm{q}_{9, \mathrm{i}}$; where ' $\mathrm{i}$ ' $=10,11,12,13$

\section{MEASURES OF COVID-19 VIRUS EFFECTIVENESS}

The MTEE, $\mathbf{A}_{\mathbf{0}}$, and $\mathbf{B}_{\mathbf{0}}$ measures due to the virus are evaluated under steady state condition and utilizing $\mathrm{C}_{1}$ is the initial (base) state.

\section{Mean time to Epidemic affected $\left(T_{0}\right)$ :}

Regenerative healthy states to which a human can transit (initial state ' 1 '), before entering any virus affected state are: ' $\mathrm{i}$ ' $=1,5,9$, Mean time to Epidemic affected $\left(\mathrm{T}_{0}\right)$, using RPGT is given as $\mathrm{T}_{0}=\left(\mathrm{E}_{1,1} \boldsymbol{\eta}_{1}+\mathrm{E}_{1,5} \boldsymbol{\eta}_{5}+\mathrm{E}_{1,9} \boldsymbol{\eta}_{9}\right) /\left(1-\mathrm{q}_{1,5} \mathrm{q}_{5,1}\right)$

Average Healthy Time $\left(\mathrm{A}_{0}\right)$ :

Regenerative states at which the system is available are ' $\mathrm{j} '=1,5,9, \&$ regenerative states are ' $\mathrm{i}$ ' $=1$ to 13. Average Healthy Time $\left(\mathrm{A}_{0}\right)$ using RPGT is as under

$$
\begin{aligned}
& \mathrm{A}_{0}=\left[\sum_{\boldsymbol{j}} \boldsymbol{E}_{\boldsymbol{\xi}, \boldsymbol{j}} \boldsymbol{f}_{\boldsymbol{j}} \boldsymbol{\eta}_{\boldsymbol{j}}\right] \div\left[\sum_{\boldsymbol{i}} \boldsymbol{E}_{\xi, \boldsymbol{i}} \boldsymbol{f}_{\boldsymbol{j}} \boldsymbol{\eta}_{\boldsymbol{i}}^{\mathbf{1}}\right] \\
& \mathrm{A}_{0}=\left(\mathrm{E}_{9,1} \boldsymbol{\eta}_{1}+\mathrm{E}_{9,5} \boldsymbol{\eta}_{5}+\mathrm{E}_{9,9} \boldsymbol{\eta}_{9}\right) / \mathrm{D} \\
& \text { Where } \mathrm{D}=\mathrm{E}_{1, i} \boldsymbol{\eta}_{\mathrm{i} ;} 1 \leq \mathrm{i} \leq 13 \\
& \text { Recovery Period }\left(\mathbf{B}_{\mathbf{0}}\right):
\end{aligned}
$$

\section{Recovery Period $\left(\mathrm{B}_{\mathbf{0}}\right)$ :}

Regenerative states where in which virus affected person is busy in recovery are $\mathrm{j}=2$ to $13, \&$ regenerative states are ' $\mathrm{i}$ ' $=1$ to 13 . Recovery Period $\left(\mathrm{B}_{0}\right)$ using RPGT is given as under

$$
\begin{aligned}
& \mathrm{B}_{0}=\left[\sum_{\boldsymbol{j}} \boldsymbol{E}_{\boldsymbol{\xi}, \boldsymbol{j}} \boldsymbol{\eta}_{\boldsymbol{j}}\right] \div\left[\sum_{\boldsymbol{i}} \boldsymbol{E}_{\boldsymbol{\xi}, \boldsymbol{i}} \boldsymbol{\eta}_{\boldsymbol{i}}^{\mathbf{1}}\right] \\
& \mathrm{B}_{0}=\left(\mathrm{E}_{1, \mathrm{j}} \boldsymbol{\eta}_{\mathrm{j}}\right) / \mathrm{D} ; 2 \leq \mathrm{j} \leq 13 .
\end{aligned}
$$

Assuming $\mathrm{n}_{\mathrm{i}}=\mathrm{n}(1 \leq \mathrm{i} \leq 4)$ and $\mathrm{m}_{\mathrm{i}}=\mathrm{m}(1 \leq \mathrm{i} \leq$

4) for ease of calculations and taking $m=0.10$, $0.20,0.30$ and $n=0.50,0.60 .0 .70$, we get

Mean time to Epidemic affected $\left(\mathrm{T}_{0}\right)$

From Table 3 and Graph 2, as we see in columns it is concluded that the value of $\mathrm{T}_{0}$ decreases rapidly i.e., with the increase in virus causing rates while observing along rows it is concluded that the value of $\mathrm{T}_{0}$ is not significantly increased with the increasing recovery rates, which is practically observed too.

\subsection{Average Healthy Time $\left(\mathrm{A}_{0}\right)$ :}

From Table 4 and Graph 3, as we see in columns it is concluded that the value of $\mathrm{A}_{0}$ decreases rapidly i.e., with the increase in virus causing rates while observing along rows it is concluded that the value of $A_{0}$ is not significantly increased with the increasing recovery rates, which is practically observed too.

\subsection{Recovery Period $\left(B_{0}\right)$ :}

From Table 5 and Graph 4, as we see in columns it is concluded that the value of $\mathrm{B}_{0}$ increases rapidly i.e., with the increase in virus causing rates while observing along rows it is concluded that the value of $\mathrm{B}_{0}$ decreases with

\begin{tabular}{|c|c|c|c|c|c|}
\hline $\mathbf{m}$ & n & n & 0.50 & 0.60 & 0.70 \\
\hline \multicolumn{3}{|c|}{0.10} & 3.77 & 3.63 & 3.56 \\
\hline \multicolumn{3}{|c|}{0.20} & 2.42 & 1.39 & 1.29 \\
\hline \multicolumn{3}{|c|}{0.30} & 1.45 & 1.30 & 1.23 \\
\hline
\end{tabular}
the increasing recovery rates, which is practically observed too.

\section{Illustrations}

Table 3: Mean time to Epidemic affected $\left(T_{0}\right)$ 


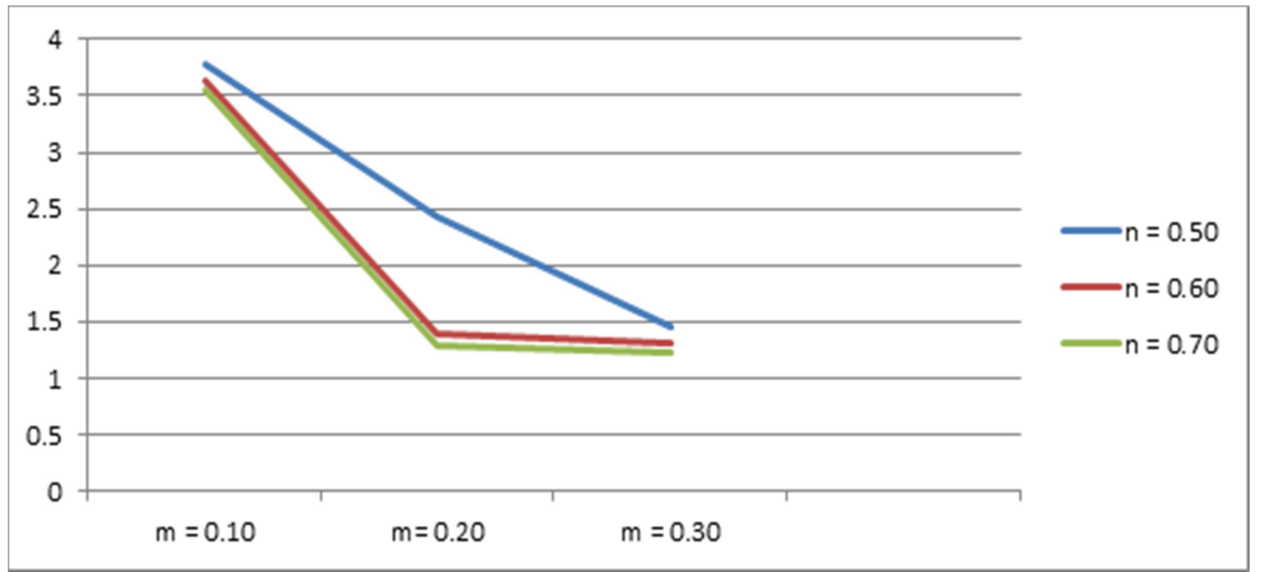

Figure 2: Mean time to Epidemic affected $\left(\mathrm{T}_{0}\right)$

Table 4: Average Healthy Time $\left(\mathrm{A}_{0}\right)$

\begin{tabular}{|c|c|c|c|c|c|}
\hline $\mathbf{m}$ & $\mathrm{n}$ & $\mathbf{n}$ & 0.50 & 0.60 & 0.70 \\
\hline \multicolumn{3}{|c|}{0.10} & 0.71 & 0.78 & 0.82 \\
\hline \multicolumn{3}{|c|}{0.20} & 0.68 & 0.75 & 0.82 \\
\hline \multicolumn{3}{|c|}{0.30} & 0.40 & 0.47 & 0.55 \\
\hline
\end{tabular}

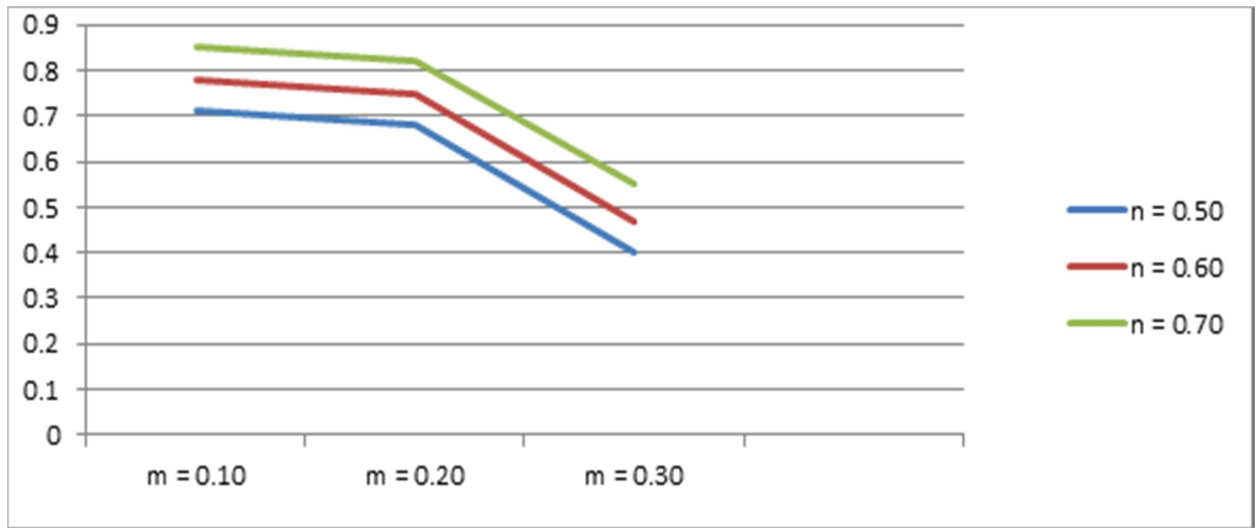

Figure 3: Average Healthy Time $\left(A_{0}\right)$

Table 5: Recovery Period $\left(B_{0}\right)$

\begin{tabular}{|c|c|c|c|c|}
\hline $\mathbf{m}$ & $\mathbf{n}$ & 0.50 & 0.60 & 0.70 \\
\hline 0.10 & & 0.31 & 0.25 & 0.21 \\
\hline 0.20 & & 0.49 & 0.44 & 0.40 \\
\hline 0.30 & & 0.69 & 0.62 & 0.59 \\
\hline
\end{tabular}

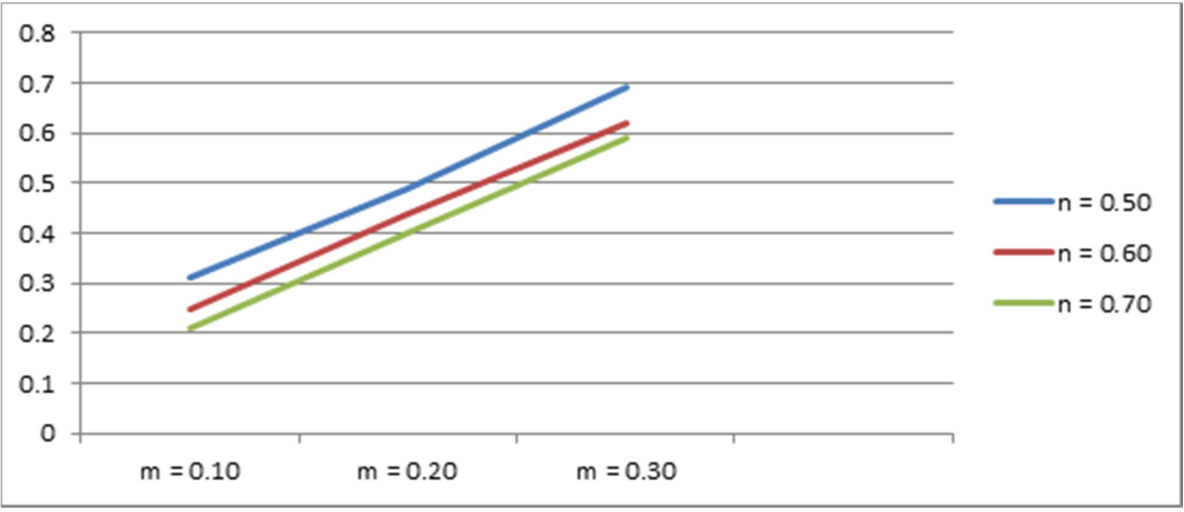

Figure 4: Recovery Period $\left(B_{0}\right)$ 


\section{CONCLUSION}

The application of RPGT has been used to discuss the transmission analysis of covid-19, in this paper three main reasons which cause positivity for covid-19 affected have been discussed, considering isolation and lockdown situations also. If rates of recovery and causes of a pandemic for a region/area are known pre hand then exact calculations can be determined applying the derived formulae in this paper. In the future there may be more factors that cause affected of covid-19 may also be included in the study.

\section{REFERENCES}

[1] Gumel A. B., Iboi E. A., Ngonghala C. N., and Elbash E. H., A primer on using mathematics to understand COVID-19 dynamics: Modeling, analysis and simulations, Infectious Disease Modelling 6, 2021, 148.

[2] Zeb A., Alzahrani E., Erturk V. S. and Zaman G.,Mathematical Model for Coronavirus Disease 2019 (COVID-19) Containing Isolation Class, BioMed Research International, 2020,2020.

[3] Singh K. and Yadav R.,Forecasting the size of Covid-19 in India using ARIMA Model, Journal of Critical Review, 7(17), 2020, 609 .

[4] Cao J. L., Hu X. R., Tu W. J. and Liu Q., Clinical features and short-term outcomes of 18 patients with corona virus disease 2019 in intensive care unit, Intensive Care Medicine, 46(5),2020, 851.

[5] Singh K. and Yadav R.,Estimation through the SEIRD Model for Covid19, TEST Engineering
Management,83.2020,24379.

[6] Zhu N., Zhang D. and Wang W., A novel coronavirus from patients with pneumonia in China, 2019, The New England Journal of Medicine, 382(8), 2020,727.

[7] Ghostine R., Gharamti M., Hassrouny S. and Hoteit I.,An Extended SEIR Model with Vaccination for Forecasting the COVID-19 Pandemic in Saudi Arabia Using an Ensemble Kalman Filter Mathematics 9(6), 2020,636.

[8] Sarkar K., Khajanchi S., Nieto J. J.,Modeling and forecasting the COVID19 pandemic in India,Chaos Solitons Fractals, 139, 2020,110049.

[9] Singh K. and Yadav R.,An epidemic model to predict the effect of infection on population of India by covid-19 corona virus, Plant Archives, 20, 2020, 1561.

[10] Kumar A., Garg D., and Goel P.,Mathematical modeling and behavioral analysis of a washing unit in paper mill, International Journal of System Assurance Engineering and Management,10, 2019,1639 .

[11] Kumar A., Goel P. and Garg D., Behaviour analysis of a bread making system, International Journal of Statistics and Applied Mathematics, 3, 2018, 56.

[12] Kumar A., Garg D. and Goel P.,Mathematical modeling and profit analysis of an edible oil refinery industry, Airo International Research Journal,8, 2017,1-8. 